\title{
Classification of ECG Recordings with Neural Networks Based on Specific Morphological Features and Regularity of the Signal
}

\author{
Katarzyna Stępien ${ }^{1}$, Iga Grzegorczyk ${ }^{1}$ \\ ${ }^{1}$ Faculty of Physics, Warsaw University of Technology, Warsaw, Poland
}

\begin{abstract}
The problem of detecting and analysing various kinds of pathologies in ECG signals has been profoundly researched in last years, but there is still no satisfying solution to distinguish such signals from normal or too noisy recordings.

Our approach to solve this problem is based on analysis of ECG signals in time and frequency domain. It combines machine learning (neural networks, bagged trees) with standard methods of classification of cardiac arrhythmias and other pathologies, from which the features for the network are determined. We want to underline that all of the features used in classifying algorithm are based on signals morphology and other physiologically reasonable factors. We concentrate mostly on features resulting from comparison of $Q R S$ complex shapes and regularity of $R R$ intervals.

In presented approach, we decided not to feed the network with many random features, but to focus only on those adequate for ECG signal analysis (in other words, only those that can be easily understood by the physician).
\end{abstract}

\section{Introduction}

Atrial Fibrillation (AF) is the most common and most complex chronic arrhythmia [1]. The mechanisms responsible for AF are still not fully understood and the treatment is very complicated. A great deal of research about this arrhythmia has been produced, but despite such effort, detection of AF episodes is still a problematic issue for both physicians and signal analysis experts. In recent years the rapid growth of artificial intelligence and machine learning techniques was noticed and they became the golden standard for solving classification problems in all areas of science, signal analysis included.

This year's PhysioNet/Computing in Cardiology Challenge 2017 is another attempt to help cardiologists in this topic. Its purpose is to correctly classify ECG signals from one lead. Each signal belongs to one of four groups: normal, AF, other (signals that have other disease, but the diagnosis is not atrial fibrillation) and noisy (the quality of which does not allow for clear diagnosis of the signal).

We analysed over 8500 ECG recordings from the PhysioNet Challenge 2017 training set. All information about the database and challenge rules can be found in [2]. Our algorithm consists of 3 parts: general conditions, neural network with 25 features and bagged tree with 14 features. To assure that our network will learn only on good quality signals, we have rejected a part of signals after preliminary analysis based only on neural network.

\section{Data}

The signals analysed in this paper were ECG recordings of duration between 9 and 60 seconds from the PhysioNet Challenge 2017 training set [2]. The recordings were labelled as normal, atrial fibrillation, other and noisy. The sampling frequency equalled $300 \mathrm{~Hz}$.

\section{Methods and Results}

\subsection{Preparation of the database}

DATASET A The training set provided by organizers was highly imbalanced and some groups were few times bigger than the others. As neural networks were our method of choice, we had to balance the set before training procedure to minimize resulting bias. We decided to add some surrogate data to noisy and atrial fibrillation recordings by taking parts of signals already available. To avoid incorrect labelling, for all of the new time series we verified whether they included crucial noisy/AF part of the signal in it. At that point number of normal recordings still outnumbered the others significantly, so we have chosen a representative group of about 2000 recordings for the training purposes. At the end, this dataset consisted of over 7000 recordings.

DATASET B Moreover, some of the recordings were provided with the wrong label. As we find it very important, to teach algorithms on proper labels, we decided to choose only those recordings from training set where there was no big doubt that the labels were assigned 
correctly. The fastest way to do so, minding the size of the dataset, was to initially train a neural network with extracted features and compare results produced by our algorithm with original labels. Looking at the plots we confirmed that our good answers belonged to the properly labelled signals. This way we had only recordings with labels we agreed with. This was less time consuming than going through all plots and changing labels manually. In this dataset, we had about 4000 signals total.

\subsection{Pre-processing of the signals \& Features}

First step of signal preparation included detecting locations of R-peaks in ECG signal, using algorithm described in $[3,4]$, as many of our features were based on regularity of QRS complexes occurrence. Because some of the features required specific filtration, we did not filter signal as a general pre-processing step, but individually for each feature. List of used features is presented below.

- $\quad$ Pow1 - power of signal filtered in bandpass 1-40 $\mathrm{Hz}$

- Pow2 - power of signal filtered in bandpass 1-3 $\mathrm{Hz}$

- Sum-change - it gives information how big is the change between base signal and signal filtered with moving mean algorithm, which corresponds to smoothness of the recording. It is calculated as sum of absolute values of differentiated signal, divided by that sum for filtered signal (see Figure $1)$.

- Angle - the number of QRS complexes in the whole signal, that are wider that double mean angle in the whole signal. It is not normalized by length of the signal.

- Zero-line - parameter determining how many times signal crosses zero line, normalized by length of signal. The bigger value is obtained, the nosier signal is.

- QRS-no - number of QRS complexes in the signal, normalized by length of signal.

- Diff-RR - mean of vector of absolute values of differences between consecutive RR intervals.

- HR-mean - average RR interval.

- HR-mean-cut - averaged RR interval, but with minimal and maximal $R R$ rejected. It is calculated to avoid some mistakes caused by incorrect QRS
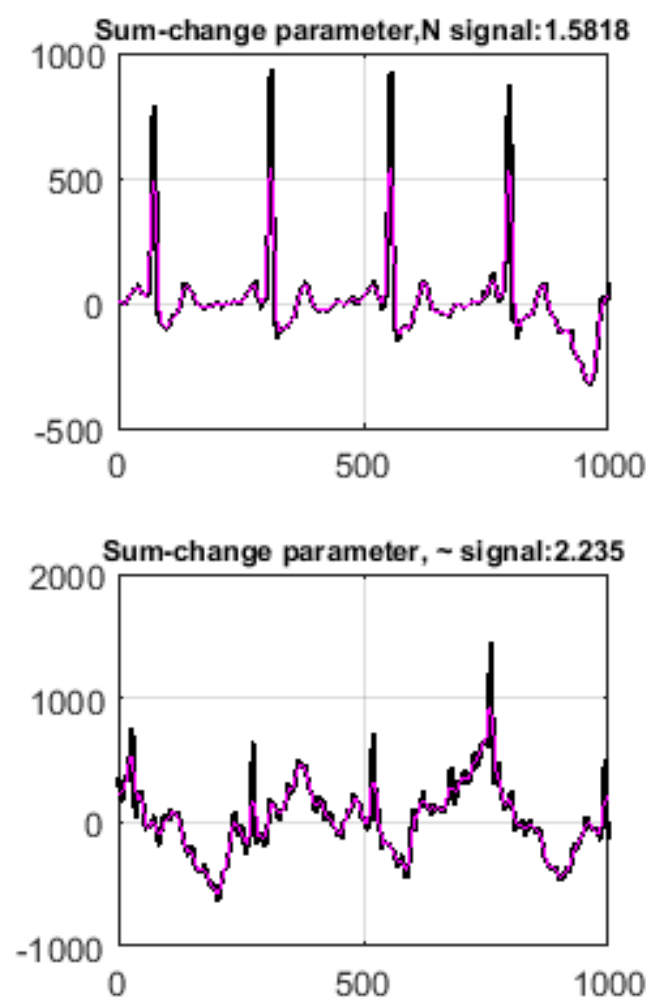

Figure 1 Sum-change parameter correspond to the information about the difference between base and filtered signal. In the first picture, normal signal is presented with the value of sum-change about 1.58. Noisy signal in the second picture is described with bigger value of this parameter (about 2.24).

detection (e.g. very long, not physiological RR intervals).

- Group-no - number of groups of different complex shapes in the same recording. This parameter was created by overlapping all QRS complexes in the signal. According to the physiology of myocardial activity, shapes of the consecutive peaks should be similar in a normal, healthy case. Another situation may indicate arrhythmia or other heart problem (see Figure 2). Clustering of QRS complexes is a method commonly used in analysis of medical time series, e.g. it was applied to ECG analysis by Martin Lagerholm et al. [5] and to electrogram signals by Faes et al. [6].

- Group-max - the number of complexes in the largest group divided by the number of all complexes (see Figure 2). 


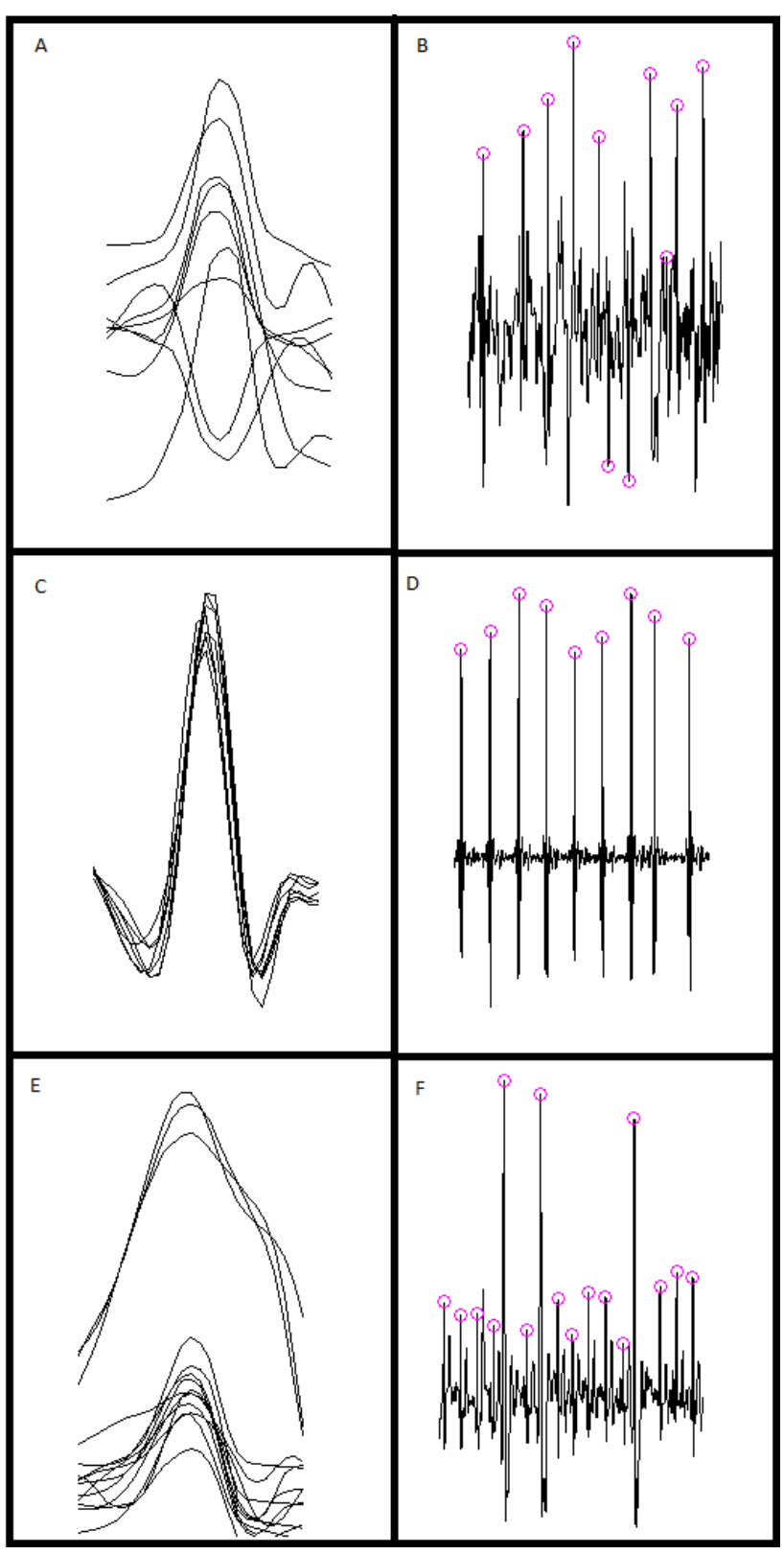

Figure 2 On the right signals with R-peaks locations are shown, on the left overlapped QRS complexes. A\&B present $\mathrm{AF}$ signal, $\mathrm{C} \& \mathrm{D}$ normal signal and $\mathrm{E} \& \mathrm{~F}$ signal labelled as other. Analyzing groups of QRS complexes in those examples, we see that for normal signal we obtain one group, for other -two groups, and 3 groups for $\mathrm{AF}$ signal. Max-group parameter for $\mathrm{C} \& \mathrm{D}$ is 1 , for $\mathrm{E} \& \mathrm{~F}$ about 0.8 , and for $\mathrm{A} \& \mathrm{~B}$ about 0.7 . The bigger this parameter is, the higher probability that signal is labelled as normal or other.
- $\quad$ STD-RR - standard deviation of RR intervals.

- Parameters given by authors of sample entry dedicated by AF identification (OriginCount, IrrEv, PACEv, AFEv).

We used not only the features from the list, but also new ones based on them. New features were created as multiplication or division for feature couples from the list. To choose the best set of features, we used brute-forced code for particular parts of algorithm (in every part another set was used).

\subsection{Classification}

\section{PRELIMINARY CONDITIONS}

The first stage of the classification in proposed algorithm are preliminary conditions, chosen empirically from the set of prepared features. It was designed to preclassify noisy and atrial fibrillation recordings. The implementation determining noisy signals includes 8 specific conditions with thresholds impossible to fulfil by a properly registered recording. The condition for AF class is based on the feature proposed by the organizers.

\section{NEURAL NETWORK CHARACTERIZATION AND TRAINING}

The neural network we created consists of one hidden layer with 13 neurons. The size of input layer is equal to number of chosen features, which is 25 . There are 4 neurons in the output layer, each of them informs what is the probability that the signal belongs to consecutive classes. At this point we choose the maximum value, which informs to which class the recording was assigned by the neural network. If it exceeds the threshold of 0.9 we take it as a final result, otherwise the problem is passed to bagged trees algorithm. This way, the result from the neural network is given with very high specificity. The neural network was trained on the dataset B, so only on the signals with proper labels.

\section{BAGGED TREES}

When the neural network described above cannot classify the signal with high certainty, the bagged trees algorithm takes the role of final classifier. It is built from 15 trees and makes decision basing on 14 carefully chosen features. This algorithm was trained on dataset A, which is a balanced set of recordings from original training set. The reason why we decided to use this method as an additional classifier was its long-time of calculations, particularly in comparison to neural networks. 


\section{Results}

Before choosing the final shape of our algorithm we run it on the validation set with preliminary conditions and neural network or bagged trees. The results of such calculations and those for the final version of the algorithm are presented in the Table 1.

Table 1. Partial results for validation set.

\begin{tabular}{lccc}
\hline Label $\backslash$ method & $\begin{array}{c}\text { Neural } \\
\text { Networks }\end{array}$ & $\begin{array}{c}\text { Bagged } \\
\text { trees }\end{array}$ & $\begin{array}{c}\text { Final } \\
\text { version }\end{array}$ \\
\hline Normal & 0.85 & 0.88 & 0.86 \\
Atrial Fibrillation & 0.76 & 0.96 & 0.82 \\
Other & 0.55 & 0.82 & 0.67 \\
Noisy & 0.72 & 0.78 & 0.78 \\
F1 score & $\mathbf{0 . 7 2}$ & $\mathbf{0 . 8 9}$ & $\mathbf{0 . 7 9}$ \\
\hline
\end{tabular}

It can be seen that the neural network had a big problem with proper classification of recordings from group other. It might be a consequence of incorrect labelling in the validation set. The score obtained for the bagged trees is much higher, but might result from the bigger data set on which it was trained and better compatibility with validation set labels. That is why above algorithms were combined in the final solution. The final score in PhysioNet Challenge 2017 equalled 0.75 in $\mathrm{F} 1$ value. Results for the test set were: 0,90 - normal, 0,70 - atrial fibrillation, 0,64 - other and 0,44 - noisy. Results for the training set were as follows: 0.79 in F1 value, 0.89 normal, 0,78 - atrial fibrillation, 0,70 - other and 0,58 noisy.

\section{Discussion and conclusions}

Proposed algorithm for ECG signals classification consisted of 3 main components: general conditions, neural network with 25 features and bagged trees algorithm with 14 features. The selected machine learning tools were trained on different, specially prepared datasets of balanced structure. Also, number of features chosen in each step varied to make it more efficient.

Results obtained with described algorithm show that classifying normal recordings was the easiest task, while the most problematic to classify correctly were signals with label 'other'. We presume this is because sometimes similarities between this two groups were impossible to determine without making algorithm oversensitive to singular changes in QRS complex modifications.

Although the algorithm performs sufficiently, its' effectiveness might be improved by increasing the complexity of the neural network.

\section{Acknowledgements}

The authors would like to thank Faculty of Physics of Warsaw University of Technology for the support. Special thanks go to the head of Cardiovascular Physics Group Professor Jan Żebrowski. We would also like to thank Assistant Professor Anna Wróblewska from Faculty of Mathematics \& Information Science of Warsaw University of Technology for consultations and substantive support.

\section{References}

[1] Omer Brenfeld, Jose' Jalife Complex Fractionated Atrial Electrograms. Is This the Beast to Tame in Atrial Fibrillation? Circ Arrhythm Electophysiol. 2011; 4:426-428.

[2] Gari Clifford, Chengyu Liu, Benjamin Moody, Ikaro Silva, Qiao Li, Alistair Johnson, Roger Mark, AF Classification from a Short Single Lead ECG Recording: the PhysioNet Computing in Cardiology Challenge 2017, Computing in Cardiology (Rennes: IEEE), Vol 44, 2017 (In Press)

[3] Behar Joachim, Jonhson Alistair, Clifford Gari D., Oster Julien A Comparison of Single Channel Foetal ECG Extraction Methods. Annals of Biomedical Engineering. 42(6), 1340-53. 2014.

[4] Johnson, A. E., Behar, J., Andreotti, F., Clifford, G. D., \& Oster, J. (2014, September). R-peak estimation using multimodal lead switching. In Computing in Cardiology Conference (CinC), 2014 (pp. 281-284). IEEE.

[5] Lagerholm, Martin, et al. "Clustering ECG complexes using Hermite functions and self-organizing maps." IEEE Transactions on Biomedical Engineering 47.7 (2000): 838848.

[6] Faes, Luca, et al. "A method for quantifying atrial fibrillation organization based on wave-morphology similarity." IEEE Transactions on Biomedical Engineering 49.12 (2002): 1504-1513.

Address for correspondence.

Iga Grzegorczyk,

Warsaw University of Technology, Faculty of Physics Koszykowa 75 00-662 Warsaw, Poland

igagrze@gmail.com 